\title{
Specificity of ADAR-mediated RNA editing in newly identified targets
}

\author{
EVA M. RIEDMANN, ${ }^{1}$ SANDY SCHOPOFF, ${ }^{1}$ JOCHEN C. HARTNER, ${ }^{2,3}$ and MICHAEL F. JANTSCH ${ }^{1}$ \\ ${ }^{1}$ Department of Chromosome Biology, Max F. Perutz Laboratories, University of Vienna, A-1030 Vienna, Austria \\ ${ }^{2}$ Max-Planck-Institute for Medical Research, 69120 Heidelberg, Germany
}

\begin{abstract}
Adenosine deaminases that act on RNA (ADARs) convert adenosines to inosine in both coding and noncoding double-stranded RNA. Deficiency in either ADAR1 or ADAR2 in mice is incompatible with normal life and development. While the ADAR2 knockout phenotype can be attributed to the lack of editing of the GluR-B receptor, the embryonic lethal phenotype caused by ADAR1 deficiency still awaits clarification. Recently, massive editing was observed in noncoding regions of mRNAs in mice and humans. Moreover, editing was observed in protein-coding regions of four mRNAs encoding FInA, CyFip2, BIcap, and IGFBP7. Here, we investigate which of the two active mammalian ADAR enzymes is responsible for editing of these RNAs and whether any of them could possibly contribute to the phenotype observed in ADAR knockout mice. Editing of Blcap, FlnA, and some sites within B1 and B2 SINEs clearly depends on ADAR1, while other sites depend on ADAR2. Based on our data, substrate specificities can be further defined for ADAR1 and ADAR2. Future studies on the biological implications associated with a changed editing status of the studied ADAR targets will tell whether one of them turns out to be directly or indirectly responsible for the severe phenotype caused by ADAR1 deficiency.
\end{abstract}

Keywords: ADAR; RNA editing; mouse SINEs; substrate-specificity

\section{INTRODUCTION}

Adenosine deaminases that act on RNA (ADARs) convert adenosines to inosines in structured and double-stranded RNA (dsRNA). In mammals, RNA editing was first observed in mRNAs encoding proteins of the central nervous system. Since inosine is interpreted as guanosine during translation, editing within a coding region will often lead to functional alterations of the encoded protein (Maas et al. 2003). Widespread editing of nontranslated RNA species or noncoding regions of RNA transcripts has also been described (Athanasiadis et al. 2004; Blow et al. 2004, 2006; Levanon et al. 2004). This suggests that other aspects of RNA function, including splicing, localization, translation, and transcript stability may also be affected by RNA editing.

\footnotetext{
${ }^{3}$ Present address: Department of Pediatric Oncology, Dana-Farber Cancer Institute, Harvard Medical School, 44 Binney Street, M654, Boston, MA 02115, USA.

Reprint requests to: Michael F. Jantsch, Department of Chromosome Biology, Max F. Perutz Laboratories, University of Vienna, Dr. Bohr Gasse 1, A-1030 Vienna, Austria; e-mail: michael.jantsch@univie.ac.at; fax: 43-1-4277-9562.

Article published online ahead of print. Article and publication date are at http://www.rnajournal.org/cgi/doi/10.1261/rna.923308.
}

In mammals, three ADAR proteins and their corresponding genes have been identified (O'Connell et al. 1995; Melcher et al. 1996a,b; Chen et al. 2000). While ADAR1 is ubiquitously expressed, ADAR2 expression levels are highest in the brain. Both enzymes mediate A-to-I conversion in synthetic dsRNAs and naturally occurring substrates. ADAR3, exclusively expressed in the brain, has so far not shown any catalytic activity using synthetic dsRNA or known ADAR targets.

The two active enzymes ADAR1 and ADAR2 have different, but sometimes overlapping target specificities, as can be concluded from in vitro studies investigating the glutamate receptor (gluR) and the 5-HT2C serotonin receptor pre-mRNAs. For instance, either ADAR1 or ADAR2 can edit the GluR-B R/G site (Melcher et al. 1996b), and the serotonin A and C editing sites (Burns et al. 1997). In contrast, the serotonin $\mathrm{B}$ site is deaminated exclusively by ADAR1, while the GluR-B Q/R and the serotonin D sites are deaminated only by ADAR2 (Melcher et al. 1996b; Burns et al. 1997; Yang et al. 1997). The selectivity of the two enzymes was also shown in vivo. An almost complete lack of editing at the serotonin receptor A and B sites was noted in transcripts derived from ADAR1 knockout cell lines (Hartner et al. 2004; Wang et al. 2004). In contrast, 
editing of the GluR-B Q/R site was unchanged (Wang et al. 2004).

The basis for their distinct, but overlapping specificities, is not well understood. Like most dsRNA binding proteins, ADAR1 and ADAR2 will bind to any dsRNA without sequence specificity. However, ADARs deaminate certain adenosines more efficiently than others. It has been proposed that the structure of the RNA substrate rather than its sequence might determine selectivity (Bass 1997). While ADARs deaminate perfectly base-paired dsRNA promiscuously, selective deamination is observed in dsRNAs containing mismatches, bulges, and internal loops (Polson and Bass 1994). Internal loops delineate helix ends for ADAR1, and adenosines close to the $3^{\prime}$ termini of such helices are less likely to be edited (Lehmann and Bass 1999). ADAR1 and ADAR2 are reported to have distinct, but sometimes overlapping specificities. ADAR1 has a $5^{\prime}$ neighbor preference $(A=U>C>G)$ but no apparent 3' neighbor preference (Polson and Bass 1994). The $5^{\prime}$ neighbor preference of $\operatorname{ADAR} 2(A \approx U>C=G)$ is similar to that of ADAR1, but in addition, ADAR2 also shows a $3^{\prime}$ neighbor preference ( $\mathrm{U}=\mathrm{G}>\mathrm{C}=\mathrm{A})$ (Lehmann and Bass 2000). However, some adenosines fail to be edited, even though they appear to be in the right context. Apart from the surrounding sequence, editing efficiency is strongly influenced by the base opposing the edited adenosine. A:C mismatches are preferred over A:A, A:G mismatches, or A:U base pairs (Wong et al. 2001).

Editing of transcripts by ADAR enzymes is essential for normal life and development. Mice deficient in ADAR2 develop normally, but are prone to epileptic seizures and die young. The impaired phenotype seems to result entirely from a single under-edited position, namely, the $Q / R$ site in the GluR-B transcript, which is normally edited to almost $100 \%$. Full phenotypic rescue is achieved by introducing a GluR-B in the edited version (Higuchi et al. 2000). The phenotype of mice deficient in ADAR1 is more severe. ADAR1-deficient mice develop a severe liver defect, involving cells of both the hematopoietic and hepatic lineages, and die between E11.5 and 12.5. Thus, ADAR1 seems to play a critical role in non-nervous tissue, which is likely to include transcript editing (Hartner et al. 2004).

Since ADAR $1^{-l-}$ lethality could not be explained by any of the known editing events, the search for novel targets is in progress. A number of groups have used computational analysis to identify thousands of A-to-I editing sites in the human transcriptome (Athanasiadis et al. 2004; Blow et al. 2004; Kim et al. 2004; Levanon et al. 2004). Most of these sites reside in Alu elements located in untranslated regions (UTRs), introns, and intergenic regions. Alu repeats of reversed orientation can base pair to form the dsRNA structure required for targeting by ADARs. The abundance of Alu elements in the genome explains the high editing levels observed in these repeats. A recent study has elucidated the RNA-editing levels in mouse repeats (Neeman et al. 2006).
Here, RNA editing is determined by specific properties of different repeat families such as abundance, length, and divergence. Editing sites in mouse are found in different types of repeats, mainly the SINEs B1 and B2, the L1 LINE, and the MalR LTR (Neeman et al. 2006).

Aside from editing in noncoding regions, four novel human substrates have been identified using comparative genomics and expressed sequence analysis (Levanon et al. 2005). Interestingly, none of the found substrates-Blcap, $I g f b p 7, C y f i p 2$, and $F \ln A$-encodes a receptor protein, but the latter two are strongly expressed in the central nervous system and seem to be involved in proper nervous system function. Blcap (bladder cancer-associated protein) is differentially expressed in bladder cancer (Gromova et al. 2002) and renal cancer cell lines (Rae et al. 2000) and is predicted to encode a small globular protein with two transmembrane helices. Editing is responsible for recoding of the second amino acid of the final protein from Y/C (Levanon et al. 2005). Further downstream, two additional recoding editing events $(Q / R$ and $K / R)$ were identified in another screen (Clutterbuck et al. 2005). The CYFIP2 (cytoplasmic FMR1 interacting protein 2) protein is highly conserved in both invertebrates and vertebrates. A single editing event resulting in a K/E substitution was identified in the CYFIP2 transcript and editing was most pronounced in brain (Levanon et al. 2005). In addition to cross-linking actin, the $280-\mathrm{kDa}$ protein Filamin A (FlnA) has been reported to interact with a variety of cellular proteins of diverse function (Popowicz et al. 2006). Thus, FlnA constitutes a versatile molecular scaffold for cell motility and signaling. The single editing site identified in FlnA leads to a Q/R substitution and is located in a region with many potentially interesting binding partners (Levanon et al. 2005).

In the present study we set out to ascertain whether any of the novel identified editing events contribute to the unexplained ADAR $1^{-l-}$ phenotype. Possible target preferences of ADAR1 and ADAR2 were examined by determining the editing status of transcripts derived from mice deficient in either enzyme. We looked at the novel coding targets Cyfip2, Blcap, and $F \ln A$, as well as two examples each for mouse B1 and B2 SINEs.

\section{RESULTS}

\section{Editing status of coding targets in mice deficient in either Adar1, Adar2, or both}

The coding targets Blcap, Cyfip2, and $F \ln A$ were analyzed with respect to which of the ADAR enzymes is responsible for editing. The regions harboring adenosines subjected to editing were amplified from cDNAs derived from mice deficient in ADAR2. To determine the impact of ADAR1 on the editing of these substrates, cDNAs of neuronal cultures derived from ADAR1 or ADAR1/ADAR2-deficient embryos were used. Lack of the responsible enzyme should lead to a 
decrease of editing at a particular site. Indeed, for BLCAP the lack of ADAR1 results in a decrease of editing at the predicted Y/C site (Table 1). However, a low editing level is still seen, indicating that ADAR2 can partially take over the function of ADAR1 at this particular position. A second editing site $(\mathrm{Q} / \mathrm{R})$ just downstream from the $\mathrm{Y} / \mathrm{C}$ site shows a similar pattern. Editing levels drop to zero in cells derived from neuronal cultures deficient in both ADAR1 and ADAR2.

In the case of CYFIP2, the enzyme responsible for editing seems to be ADAR2. While a lack of ADAR1 does not change editing levels, the absence of ADAR2 results in a significant decrease (Table 1). Again, however, editing levels do not drop to zero. This is only the case in cells deficient in both ADAR1 and ADAR2.

Finally, the results obtained for FLNA are not as distinct as for the two coding targets mentioned above. There is only a slight decrease of editing at the predicted site $(\mathrm{Q} / \mathrm{R})$ in the absence of ADAR2. Lack of ADAR1 does not have an effect (Table 1). Editing levels never drop to zero, but are generally rather low. No editing at all could be seen in FLNA fragments amplified from cDNAs derived from cells deficient in both ADAR1 and ADAR2, indicating a joint role of these two enzymes in the editing of FLNA.

\section{Editing status of noncoding targets in mice deficient in either Adar1, Adar2, or both}

\section{B1 SINES}

The EXOC8 transcript harbors three B1 repeats in close vicinity in its $3^{\prime} U T R$. The repeat located in the middle is of inverted orientation, and folding predictions show that it forms a dsRNA structure with the repeat located downstream. The three B1 repeats were amplified from cDNA derived from mice deficient in either ADAR1 or ADAR2. The resulting PCR products were bulk sequenced and analyzed for A-to-G mismatches at the predicted sites. Editing events occurred in both repeats that were predicted to

\begin{tabular}{|c|c|c|c|c|}
\hline Tissue & & $\begin{array}{l}\text { Blcap } \\
\text { UAUU }\end{array}$ & $\begin{array}{c}\text { Cyfip2 } \\
\text { UAAA }\end{array}$ & $\begin{array}{l}F \ln A \\
\text { CAG }\end{array}$ \\
\hline $\mathrm{NC}$ & $\mathrm{ADAR}^{+/+}, \mathrm{ADAR}^{+/+}$ & 36.5 & 54.5 & 4.5 \\
\hline NC & $\mathrm{ADAR}^{-1-}, \mathrm{ADAR}^{+/+}$ & 8.5 & 50 & 5 \\
\hline NC & ADAR $^{-/-}$, ADAR2 $^{-/-}$ & 0 & 0 & 0 \\
\hline Brain & $\mathrm{ADAR}^{1+/+}, \mathrm{ADAR}^{+/+}$ & 50 & 90 & 16.5 \\
\hline Brain & $\mathrm{ADAR}^{+/+}, \mathrm{ADAR}^{-1-}$ & 33.5 & 11 & 13.5 \\
\hline
\end{tabular}

form a dsRNA structure, but were not observed in the unpaired repeat located upstream (Table 2). Editing could be verified for five (Table 2, sites 1-4, 6) out of seven sites predicted for the two repeats. Two predicted editing sites showed no editing in brain or primary neuronal culture. Further, our experimental approach identified three additional editing sites in the forward repeat (Table 2, sites $5,7,8)$. While a lack of ADAR2 does not decrease editing at sites 1,2 , and 6 , editing levels drop to zero at these sites in ADAR1 knockout mice, demonstrating their editing by ADAR1 (Table 2). In contrast, site 5, which is edited to $100 \%$ in primary neuronal culture, but shows zero editing in brainderived transcripts, is not at all affected by a lack of ADAR1. In the absence of ADAR2, editing at site 3 was significantly lower, or in the case of site 8 , no longer detectable, thus making these sites look like preferential ADAR2 targets. Deamination of the remaining sites (Table 2, sites 4,7) seems to be affected by the lack of either ADAR, suggesting a joint role of ADAR1 and ADAR2 in editing these sites.

Further, editing has been investigated in the CD300a transcript. The two $\mathrm{B} 1$ repeats, located in $\mathrm{CD} 300 \mathrm{a}$, are predicted to form a duplex and are both edited at six positions (Table 3). The rather poor expression levels of this cDNA precluded investigation by bulk sequencing. Therefore, to correct for contaminating PCR species, editing levels were determined by sequencing of cloned products. Editing at sites 7, 11, and 12 is completely inhibited in the absence of ADAR2. Site 10 is the only site where editing is completely inhibited in the absence of ADAR1. The majority of editing sites in the CD300a transcript, namely, the eight remaining sites, cannot clearly be assigned to one of the two enzymes. For many of them, the loss of ADAR2 has no negative effect on editing in the brain. Six out of 12 editing sites are not deaminated in transcripts derived from primary neuronal culture.

\section{B2 SINES}

The mouse GAB2 gene harbors two closely spaced B2 elements in an intron. These two inverted repeats are separated by less than 40 nucleotides (nt), allowing us to observe editing in both strands simultaneously. The region of interest was amplified from cDNA derived from mice or cells lacking either ADAR1 and/or ADAR2. Two novel editing sites (Table 4 , sites 3,4 ) were identified experimentally in addition to the three predicted sites (Table 4,1,2,5). Site 1 is highly edited in brain and neuronal culture (Table 4). While a lack of ADAR1 does not decrease editing at this site, editing levels drop to zero in ADAR2 knockout mice, suggesting that this site is an ADAR2 target. Similar tendencies are seen for sites 3 and 4 . On the other hand, site 2 is no longer edited in the absence of ADAR1, indicating that this site is preferentially edited by ADAR1. Site 5 seems to be affected by the lack of both, ADAR1 or ADAR2, suggesting that the two enzymes play a shared role in the editing of this site. 
TABLE 2. Editing (in \%) at individual sites of reverse (-) and forward (+) B1 elements in the Exoc8 gene as determined by bulk sequencing

\begin{tabular}{|c|c|c|c|c|c|c|c|c|c|}
\hline \multirow[b]{2}{*}{ Tissue } & & \multicolumn{4}{|c|}{ Repeat (-) } & \multicolumn{4}{|c|}{ Repeat (+) } \\
\hline & & $\begin{array}{l}\text { Site } 1 \\
\text { CAC }\end{array}$ & $\begin{array}{l}\text { Site } 2 \\
\text { UAAA }\end{array}$ & $\begin{array}{l}\text { Site } 3 \\
\text { GAUU }\end{array}$ & $\begin{array}{c}\text { Site } 4 \\
\text { AAG }\end{array}$ & $\begin{array}{c}\text { Site } 5^{\mathrm{a}} \\
\text { GAAA }\end{array}$ & $\begin{array}{l}\text { Site } 6 \\
\text { UAG }\end{array}$ & $\begin{array}{c}\text { Site } 7^{\mathrm{a}} \\
\text { CAGG }\end{array}$ & $\begin{array}{l}\text { Site } 8^{\mathrm{a}} \\
\text { UAUU }\end{array}$ \\
\hline Opposite & & C & C & C & C & $U$ & A & $U$ & C \\
\hline NC & Adar1 $^{+/+}$ & 40.5 & 71 & 0 & 27 & 100 & 70.5 & 13.5 & 0 \\
\hline NC & Adar1 $^{-/-}$ & 0 & 0 & 0 & 0 & 100 & 0 & 0 & 0 \\
\hline Brain & Adar2 $^{+/+}$ & 54 & 80.5 & 18 & 39 & 0 & 56.5 & 50 & 21.5 \\
\hline Brain & Adar $^{-/-}$ & 50 & 76.5 & 3.5 & 15 & 0 & 66 & 0 & 0 \\
\hline
\end{tabular}

The surrounding and neighboring nucleotides are indicated. The sites shown here are also displayed in Figure 3 and Supplemental Figure 1. (NC) Primary neuronal culture. Results obtained by bulk sequencing.

${ }^{\mathrm{a}}$ Experimentally identified editing site.

targets seems to be responsible for the observed embryonic lethality. The identification of CYFIP2 as an ADAR2 target presumably excludes it as a candidate. However, editing of FLNA involves both ADAR1 and ADAR2, and BLCAP seems to be preferentially targeted by ADAR1.

One editing site $(\mathrm{Q} / \mathrm{R})$ has been identified in the FLNA transcript. A slight reduction in editing is seen in mice deficient in ADAR2, while a lack of ADAR1 shows no immediate effect. Yet, the fact that ADAR2-deficient mice still show a significant amount of FLNA suggests that ADAR1 can edit this RNA. The widely expressed FLNA protein

Editing has also been observed in an aberrant, incompletely spliced transcript of the ABLIM1 gene. This gene harbors two B2 repeats of inverse orientation in intronic regions. The two repeats were amplified from cDNA derived from mice deficient in either ADAR1 or ADAR2. Editing was only observed in one of the two repeats (Table 5). Sequence analysis revealed one novel editing site (2) in addition to the five predicted sites in this region. While three sites were not edited in transcripts derived from either brain or primary neuronal culture, editing of the remaining three sites (1-3) could be detected in brain. Editing levels of $10 \%-20 \%$ were observed for these sites. These levels dropped to zero in mice deficient in ADAR2, suggesting that in brain, sites 1,2, and 3 are preferentially targeted by ADAR 2 .

\section{DISCUSSION}

While the phenotype of ADAR2 knockout mice can be explained by underediting of the $\mathrm{Q} / \mathrm{R}$ site in the GluR-B transcript, the severe phenotype of ADAR1 knockout mice is still unsettled. None of the previously described coding cross-links actin filaments by its $\mathrm{N}$-terminal actin-binding domain. The rest of the protein consists of a rod-like domain of 24 repeats, interrupted by two hinge regions. It is this rod-like domain, in particular, repeats 15-24, which is involved in binding over 45 cellular proteins of various functions (Robertson 2005). While null mutations of FLNA lead to defects in neuronal migration, vascular function, and connective tissue integrity (PVNH), missense mutations in the same gene product produce a spectrum of malformations in multiple organ systems, especially the skeleton (OPD-spectrum disorders) (Feng and Walsh 2004). The $\mathrm{Q} / \mathrm{R}$ substitution in FLNA maps to repeat 22, which has been reported to bind to Glutamate receptor type 7, Calcitonin receptor, Androgen receptor, SEK-1, BRCA-2, Smad, Caveolin-1, and Integrin (Popowicz et al. 2006). Editing at this position might affect binding to one of the potential binding partners described for this region. Interestingly, the adenosine to be edited is located in the immediate vicinity to an exon-intron junction. Therefore, editing at this position is likely to influence splicing. Alteration of the binding capacity of FLNA to one of its many

TABLE 3. Editing (in \%) at individual sites of B1 elements in the CD300a gene as determined by sequencing of individual clones

\begin{tabular}{|c|c|c|c|c|c|c|c|c|c|c|c|c|c|c|c|}
\hline \multirow[b]{2}{*}{ Tissue } & & \multicolumn{7}{|c|}{ Repeat (-) } & \multicolumn{7}{|c|}{ Repeat (+) } \\
\hline & & $\begin{array}{l}\text { Site } 1^{\mathrm{a}} \\
\text { AAC }\end{array}$ & $\begin{array}{l}\text { Site } 2 \\
\text { UAA }\end{array}$ & $\begin{array}{c}\text { Site } 3^{a} \\
\text { AAA }\end{array}$ & $\begin{array}{c}\text { Site } 4 \\
\text { CAG }\end{array}$ & $\begin{array}{l}\text { Site } 5 \\
\text { CAG }\end{array}$ & $\begin{array}{c}\text { Site } 6^{a} \\
\text { AAG }\end{array}$ & $\begin{array}{c}\text { Clones } \\
\text { analyzed }\end{array}$ & $\begin{array}{c}\text { Site } 7^{\mathrm{a}} \\
\text { GAG }\end{array}$ & $\begin{array}{l}\text { Site } 8 \\
\text { CAG }\end{array}$ & $\begin{array}{l}\text { Site } 9^{a} \\
\text { UAC }\end{array}$ & $\begin{array}{c}\text { Site } 10 \\
\text { GAG }\end{array}$ & $\begin{array}{c}\text { Site } 11^{\mathrm{a}} \\
\text { CAG }\end{array}$ & $\begin{array}{c}\text { Site } 12^{\mathrm{a}} \\
\text { CAG }\end{array}$ & $\begin{array}{c}\text { Clones } \\
\text { analyzed }\end{array}$ \\
\hline Opposite & & U & U & U & U & U & U & & U & U & U & U & U & U & \\
\hline NC & Adar1 $^{+/+}$ & 0 & 0 & 0 & 0 & 100 & 0 & 1 & 0 & 14 & 57 & 14 & 0 & 0 & 14 \\
\hline NC & Adar1 ${ }^{-/-}$ & 0 & 0 & 0 & 9 & 9 & 0 & 12 & 11 & 0 & 0 & 0 & 0 & 0 & 18 \\
\hline Brain & Adar2 ${ }^{+/+}$ & 0 & 8 & 15 & 8 & 15 & 0 & 13 & 42 & 17 & 33 & 0 & 25 & 33 & 12 \\
\hline Brain & Adar2 ${ }^{-/-}$ & 18 & 9 & 18 & 9 & 55 & 9 & 12 & 0 & 0 & 6 & 0 & 0 & 0 & 18 \\
\hline
\end{tabular}

The surrounding and neighboring nucleotides are indicated. The sites shown here are also displayed in Figure 3 and Supplemental Figure 1. The number of clones analyzed for each element is given. Small sample sizes reflect difficulties in amplifying this element. (NC) Primary neuronal culture.

${ }^{a}$ Experimentally identified editing site. 
TABLE 4. Editing (in \%) at individual sites of B2 elements in the Gab2 gene as determined by bulk sequencing

\begin{tabular}{|c|c|c|c|c|c|c|}
\hline \multicolumn{3}{|c|}{ Repeat (-) } & \multicolumn{4}{|c|}{ Repeat (+) } \\
\hline Tissue & & $\begin{array}{l}\text { Site } 1 \\
\text { CAG }\end{array}$ & $\begin{array}{c}\text { Site } 2 \\
\text { AAG }\end{array}$ & $\begin{array}{c}\text { Site } 3^{\mathrm{a}} \\
\text { UAG }\end{array}$ & $\begin{array}{c}\text { Site } 4^{\mathrm{a}} \\
\text { GAA }\end{array}$ & $\begin{array}{l}\text { Site } 5 \\
\text { CAG }\end{array}$ \\
\hline Opposite & & C & $U$ & C & G & C \\
\hline $\mathrm{NC}$ & $\mathrm{ADAR}^{+/+}$ & 62.5 & 12 & 43 & 14.5 & 44.5 \\
\hline NC & $\mathrm{ADAR}^{-1-}$ & 68 & 0 & 39.5 & 14.5 & 26 \\
\hline Brain & $\mathrm{ADAR}^{+/+}$ & 77.5 & 22.5 & 60 & 23.5 & 56 \\
\hline Brain & $\mathrm{ADAR}^{-1-}$ & 0 & 38.5 & 24 & 4.5 & 22 \\
\hline
\end{tabular}

The surrounding and neighboring nucleotides are indicated. (NC) Primary neuronal culture. Results obtained by bulk sequencing. axperimentally identified editing site.

binding partners or aberrant splicing of the FLNA transcript could presumably affect various cellular processes in different tissues.

The BLCAP transcript shows editing at two positions corresponding to the second and fifth amino acid of the final protein, resulting in a $\mathrm{Y} / \mathrm{C}$ and $\mathrm{Q} / \mathrm{R}$ change, respectively. Sequence analysis of BLCAP CDNA, derived from mice deficient in one or the other ADAR enzyme, suggests that BLCAP is preferentially edited by ADAR1. The function of the highly conserved 87 aa-long protein is unknown (Gromova et al. 2002; Su et al. 2004). BLCAP is predicted to encode a small globular protein with two membrane helices (Clutterbuck et al. 2005). The identified editing sites locate to the $\mathrm{N}$-terminal region of the protein, predicted to be outside of the membrane. Further, deamination of these positions results in nonsynonymous amino acid substitutions of exposed residues (Clutterbuck et al. 2005). The edited adenosines and most of the adjacent bases are conserved across species, suggesting a fundamental role for editing in this region.

In the last few years it has become clear that recoding and altering of protein function is only one of the capacities of A-to-I editing. Its most common targets are untranslated sequences, particularly repetitive elements residing in introns and 3'UTRs. Further, certain microRNA (miRNA) precursors are subject to editing by ADARs. These findings suggest additional, currently unknown functions for RNA editing.

\section{Different sites in repetitive elements are edited by different ADARs}

Looking at editing events within two B1 repeats residing in the $3^{\prime}$ UTR of EXOC8 as well as within two B1 elements located in intronic regions of $C D 300 a$, we found that both ADAR1 and ADAR2 are involved in editing of B1 SINEs in mouse. Editing of some sites such as sites 1,2, and 6 of the EXOC8 transcript clearly depended on ADAR1 (Table 2). Other sites were targets for ADAR2 or allowed for editing by both enzymes. Only one site in CD300a could clearly be assigned to ADAR1. A few sites seem to be targets for ADAR2, but most of the sites could not clearly be assigned to either ADAR, indicating a joint role of the two enzymes.

Analysis of $\mathrm{B} 2$ editing on the basis of two $\mathrm{B} 2$ repeats located in an intron of the GAB2 gene as well as two intronically located B2 repeats of the Ablim 1 gene showed, similar to B1 editing, involvement of both ADAR1 and ADAR2. Generally, the B2 elements analyzed in the frame of this work contained more ADAR2 than ADAR1 editing sites. However, site 2 in the GAB2 transcript was identified as a clear ADAR1 target (Table 4). Further, the B1 elements investigated in this study contained more editing sites (eight for Exoc8 and 12 for $\mathrm{CD} 300 \mathrm{a}$ ) than the B2 repeats (five for Gab2 and three for Ablim). Our results showed that at least some sites located in mouse repetitive elements are exclusively edited by ADAR1.

An altered editing pattern of noncoding RNA due to ADAR1 deficiency is likely to affect various cellular processes associated with A-to-I editing of noncoding RNA. However, the precise role of editing in untranslated regions is poorly understood. It is thought to influence gene expression in several ways. (1) Inosine does not base pair with uracil but it does with cytosine; thus, the stability of RNA molecules might be influenced by creating or disrupting secondary structures. Subsequently, other dsRNA-dependent processes such as RNA interference (RNAi) could be affected. (2) Indeed, ADAR editing and the RNAi pathway have been linked (Bass 2000). (3) It has been observed that edited RNAs commonly have aberrant splicing patterns (Kim et al. 2004). Even if editing does not directly alter splice sites, base modifications of repetitive elements could change local RNA structures and inhibit the normal splicing process. Therefore, Alu-associated RNA editing has been suggested as a mechanism for marking nonstandard transcripts, not destined for translation (Kim et al. 2004). (4) Indeed, cellular machineries for processing multiple-inosine-containing RNAs do exist. It has been

TABLE 5. Editing (in \%) at individual sites of B2 elements in the Ablim1 gene

\begin{tabular}{|c|c|c|c|c|}
\hline \multicolumn{5}{|c|}{ Repeat (-) } \\
\hline Tissue & & $\begin{array}{l}\text { Site } 1 \\
\text { CA-A }\end{array}$ & $\begin{array}{c}\text { Site } 2^{a} \\
\text { AAAA }\end{array}$ & $\begin{array}{l}\text { Site } 3 \\
\text { AAUU }\end{array}$ \\
\hline Opposite & & U & U & A \\
\hline NC & $\mathrm{ADAR}^{+/+}$ & 0 & 0 & 0 \\
\hline NC & $\mathrm{ADAR}^{-1-}$ & 0 & 0 & 0 \\
\hline Brain & $\mathrm{ADAR}^{+/+}$ & 11 & 15.5 & 21 \\
\hline Brain & $\mathrm{ADAR}^{-1-}$ & 0 & 0 & 0 \\
\hline
\end{tabular}

The surrounding and neighboring nucleotides are indicated. (NC) Primary neuronal culture. Results obtained by bulk sequencing.

axperimentally identified editing site. 
reported that transcripts containing inosine, which is not normally part of RNA molecules, may be recognized and retained in the nucleus by a protein complex consisting of p54nrb, PSF, and matrin 3 (Zhang and Carmichael 2001). Scadden and Smith (2001) described a cytoplasmic ribonuclease activity that specifically cleaves inosine-containing transcripts. (5) Recent reports have implicated a new role for RNA editing in the control of miRNA biogenesis. A few studies have identified miRNA precursors as ADAR targets (Blow et al. 2004; Luciano et al. 2004; Yang et al. 2006). Editing of miRNA can affect their processing, as was shown for the pri-miRNA-142, which upon editing can no longer be processed by Drosha and is degraded by Tudor-SN (Yang et al. 2006). Depending on the location of the editing site(s), deamination can also result in inosine containing mature miRNAs, like in the case of miRNA22, where both the pri-miRNA and the mature miRNA sequence are edited (Luciano et al. 2004). A survey to detect RNA editing of miRNAs from different human tissues revealed that $6 \%$ of all miRNA transcripts analyzed were A-to-I edited (Blow et al. 2006). Many of the editing events were found in mature miRNAs and some were situated in the "seed" sequence. This theoretically allows them to silence a different set of target genes than is silenced by the unedited miRNA (Blow et al. 2006). The first evidence that edited miRNAs have a biological significance in vivo was recently provided on the basis of miRNA376a, which was found to be edited in its seed region, thereby redirecting the edited miRNA to a new set of targets (Kawahara et al. 2007). While editing of miRNAs can modulate target specificity, results from a new study add an additional layer of complexity to miRNA-mediated repression. A-to-I editing is often found in $3^{\prime}$ UTRs, and sometimes these editing positions have the potential to block miRNA target recognition (Liang and Landweber 2007). In general, however, RNA editing tends to avoid the miRNA target sites, suggesting that RNA editing in $3^{\prime}$ UTRs may be under functional constraint to avoid interrupting of miRNA target sites (Liang and Landweber 2007). Another recent report showed that some Alu elements within human mRNAs serve as miRNA targets (Smalheiser and Torvik 2006). A number of miRNAs show typical short-seed complementarity with a specific site within Alu elements that is highly conserved within $3^{\prime}$ UTRs of human mRNAs. This 6-mer core sequence (GCACUU) is also preserved in mouse B1 SINE consensus sequence, and was found to be present in B1 elements from EXOC8 and CD300a. In both cases, editing occurred close to but not within the 6-mer core sequence, thus not directly altering the target sequence for miRNAs. However, changed stability of the dsRNA structure, due to repeat editing, might impair targeting of specific mRNAs by their corresponding miRNA.

\section{Sequence preferences of ADAR1 and ADAR2}

ADARs act on dsRNA without apparent sequence-specific binding, but according to previous reports they do show certain neighbor base preferences. Obviously, the sample size of our study does not allow us to draw conclusions on a general pattern of preferences for one or the other ADAR. However, nearest-neighbor preference analysis of all substituted adenosines within the SINEs investigated in the course of this study reveals a slight excess of Cs one base $5^{\prime}$ to the edited base. However, a similar frequency of Cs can also be found $5^{\prime}$ to unedited adenosines. The $3^{\prime}$ neighboring base is preferentially a $G$, or more general, a purine (Fig. 1). Analysis of all unedited adenosines within the dsRNA structure shows that this $3^{\prime}$ purine preference is not simply due to sequence bias, as it occurs nearly twice as often in target trinucleotides than for non-edited adenosines. The excess of $\mathrm{G}$ one base $3^{\prime}$ to the edited base has also been reported by Kim et al. (2004), who looked at editing of embedded Alu elements in a statistical approach. The investigators further found certain trinucleotides to be favored (TAG, AAG) or disfavored (GAN, AAA) (Kim et al.

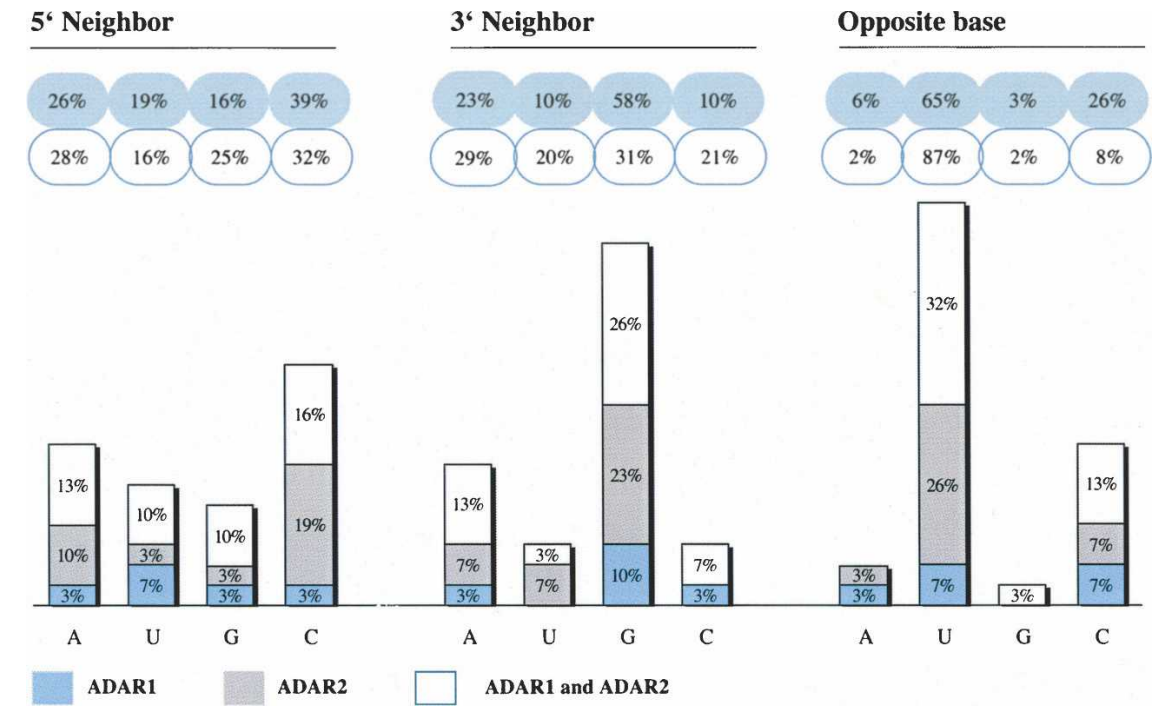

FIGURE 1. Independent nearest-neighbor preferences. Occurrence of 5'and 3 ' neighbors, as well as opposite bases were analyzed for all edited and unedited adenosines found in B1 and B2 elements. The frequency of bases neighboring the edited adenosines are indicated in blue-gray bubbles, while neighbors of unedited bases are shown in white bubbles. Strong deviation of the values between the blue and white bubbles are indicative of a sequence preference by ADARs. Each value is subdivided into sites edited exclusively by ADAR1 (indicated in light blue), ADAR2 (indicated in gray), or both enzymes (white). Opposite base preferences were only determined for adenosines clearly localized in well-base-paired regions. 
2004). Similar observations were made by Lehmann and Bass (2000). This reflects the trinucleotide found to be favored most often in our data set, UAG. Nearly $11 \%$ of all UAG trinucleotides within the dsRNA structure were edited, closely followed by the CAG and GAA triplets (about $9 \%$ and $7 \%$, respectively). UAG was found to be targeted by ADAR1 or both enzymes, but not in the group of clear ADAR2 target sites. This is in contrast to CAG and GAA trinucleotides, which displayed no clear preference for ADAR1, but for ADAR2, or both enzymes (Fig. 2). Indeed, the $\mathrm{Q} / \mathrm{R}$ site (CAG) of GluR-B has been described as an ADAR2 target in vivo (Melcher et al. 1996b) and the $Q / R$ site (CAG) of FlnA seems to also be targeted by ADAR2 (this study). Previously, the $3^{\prime} \mathrm{G}$ has been proposed as a preferred sequence context for ADAR2 (Lehmann and Bass 2000). The relatively low number of exclusive ADAR1 sites allows no general conclusion on triplet preferences for this enzyme. However, two of the six sites identified as ADAR1 targets were in the sequential context of AAG; this triplet only occurred at a very low rate within the group of exclusive ADAR2 sites (Fig. 2).

The base opposite the edited adenosine was often uracil, followed by cytosine. This is mostly due to the fact that uracil occurs most often as the opposite base. Nonetheless, the frequency of $\mathrm{C}$ opposite an edited $\mathrm{A}$ is about three times higher than expected by the natural occurrence of $\mathrm{C}$ residues opposite A residues. While edited positions in Exoc8, Gab2, and Ablim had various bases located opposite, all 12 editing sites identified in CD300a were exclusively opposed by uracil. In the Gab2 transcript, three sites

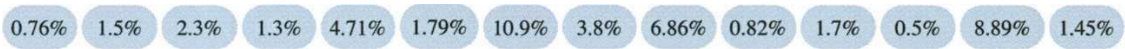

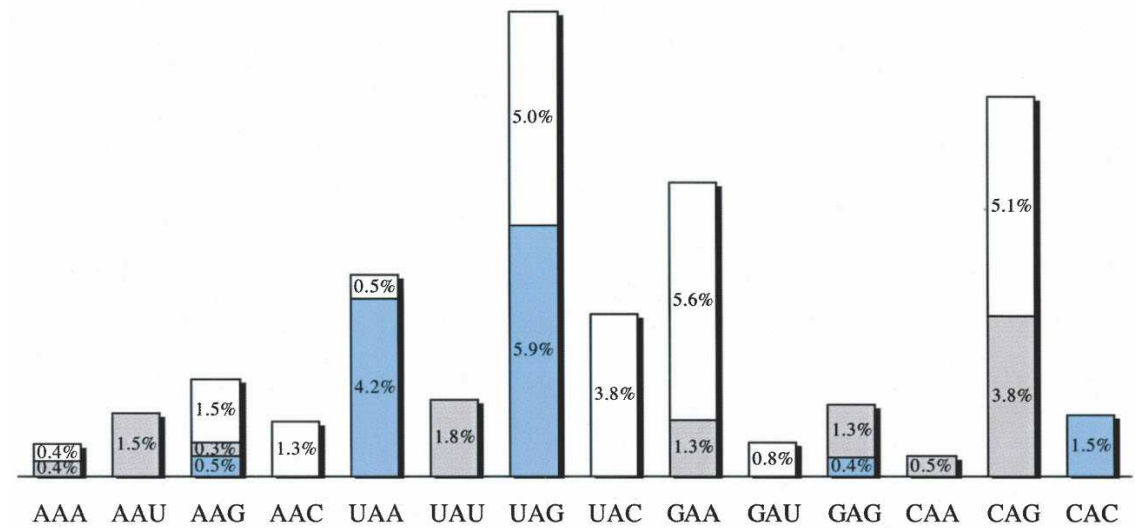

ADAR1

ADAR2

ADAR1 and ADAR2

FIGURE 2. Triplet preferences. Frequencies of triplets are shown in percent (blue-gray bubbles). Each value is subdivided into sites edited exclusively by ADAR1 (indicated in light blue), ADAR2 (indicated in gray), or both enzymes (white). To take sequence bias into account, these values represent the frequency of edited triplets corrected against their overall occurrence within the dsRNA structures. contain an A:C mismatch at the editing site, while the remaining two sites contain an A:U base pair and an A:G mismatch, respectively. The latter two sites are edited at much lower levels than the sites containing the A:C mismatch. While this is in accordance with previous results showing that an $\mathrm{A}: \mathrm{C}$ mismatch at the editing site can enhance editing by both ADARs (Wong et al. 2001), we saw comparably high levels of editing in Exoc8 at sites that contain an A:A mismatch or A:U base pair, respectively.

\section{Editing sites cluster in repeats}

Alignments between the two B1 elements or the two B2 elements examined in the frame of this study were performed in order to see whether the editing sites share any structural similarities. Interestingly, alignment of the B1 elements (CD300a and Exoc8) showed the majority of editing sites, namely 13 out of 20 , to be clustered in a relatively short region (Supplemental Fig. 1). Within this region, editing of two adenosines is conserved between the two B1 elements of CD300a and Exoc8 (Fig. 3). While editing in CD300a exclusively occurs in a perfect stem-loop, edited adenosines in Exoc8 are often found in small loops or gaps (Fig. 3). Aligning the B1 repeats with Alu shows the site 2 of Exoc8 to overlap with one of the preferred Alu editing sites (Kim et al. 2004; Levanon et al. 2004). The two studied B2 elements (Gab2 and Ablim1) are much more divergent from each other than the two B1 elements, and the editing sites are more scattered (data not shown).

In the present study we have investigated 31 editing sites, with respect to which ADAR is responsible for the A-to-I substitution. We demonstrate the involvement of ADAR1 in the editing of two novel coding targets, namely FlnA and Blcap. In addition, a handful of exclusive ADAR1 editing sites located in mouse repetitive elements B1 and B2 have been identified. Our findings add another piece to the puzzle of the yet to be explained lethal phenotype of ADAR1 knock out mice.

\section{MATERIALS AND METHODS}

\section{Editing status of coding targets}

The regions of FlnA, Blcap, and Cyfip2 that contain the recently published editing sites were amplified by PCR. As a template, cDNAs (kindly provided by Professor Peter Seeburg and Dr. Miyoko Higuchi [MaxPlanck-Institute for Medical Research]) derived from mice or embryos deficient in ADAR1, ADAR2, or both enzymes were used. These cDNAs were derived from brain of $\mathrm{ADAR}^{-/-}$mice or from primary neu- 


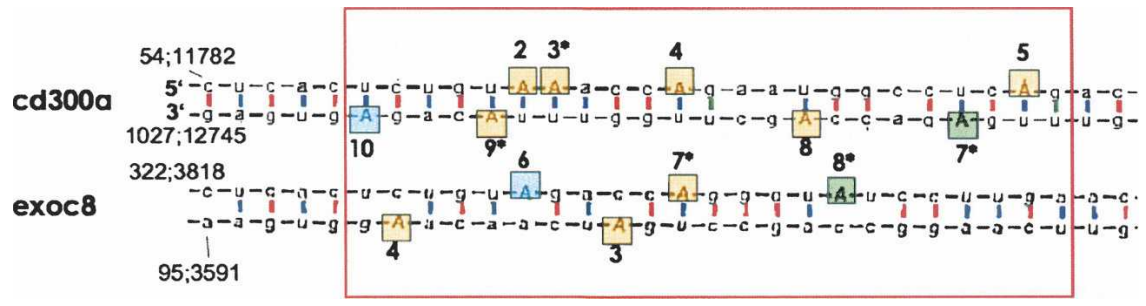

FIGURE 3. Detail of a B1 alignment. B1 elements of Exoc 8 and $C D 300 a$ were aligned. A detail from this alignment highlighting the clustering of editing events in a defined region (boxed area in Supplemental Fig. 1) is shown. Editing of two positions (sites 2 and 4 in CD300a, and 6 and 7 in Exoc8) is conserved between the two examined B1 elements. Sites edited exclusively by ADAR1 or ADAR2 are indicated in blue or green, respectively. Sites edited by either enzyme are indicated in orange. Coordinates: from accession numbers NM_198103 for Exoc8, NM_170758 for CD300a; the first number indicates the local position where the first nucleotide of the first repeat $=1$, second number indicates relative position of the repeat within the entire pre-mRNA transcript. The full alignment can be seen in the supplements.

ronal culture of ADAR $1^{-1-}$ mice and the double knockouts. In the case of FLNA primers, MJ1319 (5'-GGTGACGCCCGCCGCCTTAC-3') and MJ1317 (5'-AAGATGCTGGCTGGTTGACC-3') were designed to bind in exon 42 and exon 43. The region of interest in BLCAP was amplified using the primer pair MJ1306 (5'-GGCGTCGCCCGCCTGGGC-3') and MJ1308 (5'-GAGTGG CTGAACCACAGAGCG-3'), designed to bind in exon1 and exon2, respectively. Primers MJ1311 (5'-CCAAGAAGAGAATCA ACCTTAGC-3') and MJ1312 (5'-CTGAACAAGAGAAGTATCAC TCG $-3^{\prime}$ ) were used to PCR generate the region of Cyfip2, where editing takes place. For amplification of the fragments with Taq Polymerase (Fermentas), 30 cycles of PCR were used. The resulting products (70 base pairs [bp] for FLNA, 270 bp for $B L C A P$, and $130 \mathrm{bp}$ for CYFIP2) were isolated from a $2 \%$ agarose gel (PeqLab) and subsequently cloned into the vector pGEMTeasy. Plasmid DNA of 12 or more individual clones was isolated and sequenced using a BigDye Terminator Cycle Sequencing Kit (Applied Biosystems).

\section{Editing status of noncoding targets}

Editing of B1 SINEs was investigated on the basis of three B1 repeats located in the $3^{\prime} \mathrm{UTR}$ of the gene Exoc8 and two B1 repeats located in the gene CD300a. Editing of B2 SINEs was examined by means of two B2 repeats located in an intronic region of the Gab2 gene and two B2 repeats located in the gene Ablim1. The regions of interest were amplified by PCR from cDNAs (kindly provided by Professor Peter Seeburg and Dr. Miyoko Higuchi [MaxPlanck-Institute for Medical Research]) derived from mice or embryos deficient in either ADAR1, ADAR2, or both. The three B1 elements found in Exoc8 were amplified separately using the following primer pairs: MJ1565 (5'-CCTTAAGAATGCCAGAGA TTTG-3') and MJ1566 (5'-GCAGCACAGCATTTGAAGAGT-3') for repeat1, MJ1662 (5'-GAAACTTAGTAACTGAGTAGAG-3') and MJ1663 (5'-CCCTACTCAACAACCTGAAG-3') for repeat2, and MJ1664 (5'-CAAGGGTTTCTCTGTATAGC-3') and MJ1665 (5'-CGTTGACAAAACCCACAGC-3') for repeat3. The transcript CD300a harbors two B1 elements, which were amplified using primer pairs MJ2196 (5'-CCTAAGCTCTGAGGACAAAAGCC TG-3') and MJ2197 (5'-GGGCTACAGGCATGTAACAGACA TC-3') for repeat1 and MJ2198 (5'-GGTAGGATGATGGCTATT
TGTGACTT-3') and MJ2199 (5'-CCCACA CACCGTGGAACCTGGCTTT-3') for repeat2. In the case of Gab2, the two B2 repeats are located in close vicinity to each other and can therefore be amplified as one fragment using the primer pair MJ1857 (5' AGAGAGCTAAATAAAGTTCAGC-3') and MJ1860 (5'-CAGCTAGACTTTCACATCT AG-3'). Amplification of the two B2 repeats found in the Ablim1 gene was performed with the following primer combinations: MJ2114 (5'-CCCGCGCTCTTCATCCGAT TTC-3') and MJ2115 (5'-CCCAAGACCGT GATCCACACG-3') for repeat1, MJ2116 (5'-GGTGCCCATTTGGTCAATTCATGA$\left.3^{\prime}\right)$ and MJ2117 (5'-GGGAAACACGCTCT GATCAAT-3') for repeat2. For amplification of the fragments with Taq Polymerase (Fermentas), 30 cycles of PCR were used. The resulting products $(250-500 \mathrm{bp})$ were isolated from an agarose gel (PeqLab) and directly bulk sequenced using a BigDye Terminator Cycle Sequencing Kit (Applied Biosystems), with the exception of CD300a, which was sequenced by single-clone analysis as described for the coding targets.

\section{SUPPLEMENTAL DATA}

Supplemental material can be found at http://www.rnajournal.org.

\section{ACKNOWLEDGMENTS}

We thank Teresa Spreitzer for excellent technical assistance. Knockout cDNAs were generous gifts from Professor Peter Seeburg and Dr. Miyoko Higuchi at the Max-Planck-Institute for Medical Research in Heidelberg. This work was funded by the Austrian Science Foundation, Grant No. SFB 1706 to M.F.J.

Received November 16, 2007; accepted February 18, 2008.

\section{REFERENCES}

Athanasiadis, A., Rich, A., and Maas, S. 2004. Widespread A-to-I RNA editing of alu-containing mRNAs in the human transcriptome. PLoS Biol. 2: e391. doi: 10.1371/journal.pbio.0020391.

Bass, B.L. 1997. RNA editing and hypermutation by adenosine deamination [published erratum appears in Trends Biochem Sci 1997 Jul;22(7):278]. Trends Biochem. Sci. 22: 157-162.

Bass, B. 2000. Double-stranded RNA as a template for gene silencing. Cell 101: 235-238.

Blow, M., Futreal, P.A., Wooster, R., and Stratton, M.R. 2004. A survey of RNA editing in human brain. Genome Res. 14: 2379-2387.

Blow, M., Grocock, R., van Dongen, S., Enright, A., Dicks, E., Futreal, P., Wooster, R., and Stratton, M. 2006. RNA editing of human microRNAs. Genome Biol. 7: R27. doi: 10.1186/gb-2006-7-4-r17.

Burns, C., Chu, H., Rueter, S.M., Hutchinson, L., Canton, H., Sanders-Bush, E., and Emeson, R.B. 1997. Regulation of serotonin-2C receptor G-protein coupling by RNA editing. Nature 387: 303-308.

Chen, C.X., Cho, D.S., Wang, Q., Lai, F., Carter, K.C., and Nishikura, K. 2000. A third member of the RNA-specific adenosine deaminase gene family, ADAR3, contains both single- and double-stranded RNA binding domains. RNA 6: 755-767. 
Clutterbuck, D.R., Leroy, A., O'Connell, M.A., and Semple, C.A. 2005. A bioinformatic screen for novel A-I RNA-editing sites reveals recoding editing in BC10. Bioinformatics 21: 2590-2595.

Feng, Y. and Walsh, C. 2004. The many faces of filamin: A versatile molecular scaffold for cell motility and signalling. Nat. Cell Biol. 6: 1034-1038.

Gromova, I., Gromov, P., and Celis, J. 2002. bc10: A novel human bladder cancer-associated protein with a conserved genomic structure downregulated in invasive cancer. Int. J. Cancer 98: 539-546.

Hartner, J.C., Schmittwolf, C., Kispert, A., Muller, A.M., Higuchi, M., and Seeburg, P.H. 2004. Liver disintegration in the mouse embryo caused by deficiency in the RNA-editing enzyme ADAR1. J. Biol. Chem. 279: 4894-4902.

Higuchi, M., Maas, S., Single, F.N., Hartner, J., Rozov, A., Burnashev, N., Feldmeyer, D., Sprengel, R., and Seeburg, P.H. 2000. Point mutation in an AMPA receptor gene rescues lethality in mice deficient in the RNA-editing enzyme ADAR2. Nature 406: $78-81$.

Kawahara, Y., Zinshteyn, B., Sethupathy, P., Iizasa, H., Hatzigeorgiou, A., and Nishikura, K. 2007. Redirection of silencing targets by adenosine-to-inosine editing of miRNAs. Science 315: 1137-1140.

Kim, D., Kim, T., Walsh, T., Kobayashi, Y., Matise, T., Buyske, S., and Gabriel, A. 2004. Widespread RNA editing of embedded alu elements in the human transcriptome. Genome Res. 14: 17191725 .

Lehmann, K. and Bass, B. 1999. The importance of internal loops within RNA substrates of Adar1. J. Mol. Biol. 291: 1-13.

Lehmann, K. and Bass, B. 2000. Double-stranded RNA adenosine deaminases Adar1 and Adar2 have overlapping specificities. Biochemistry 39: 12875-12884.

Levanon, E., Eisenberg, E., Yelin, R., Nemzer, S., Hallegger, M., Shemesh, R., Fligelman, Z., Shoshan, A., Pollock, S., Sztybel, D., et al. 2004. Systematic identification of abundant A-to-I editing sites in the human transcriptome. Nat. Biotechnol. 22: 1001-1005.

Levanon, E., Hallegger, M., Kinar, Y., Shemesh, R., DjinovicCarugo, K., Rechavi, G., Jantsch, M., and Eisenberg, E. 2005. Evolutionarily conserved human targets of adenosine to inosine RNA editing. Nucleic Acids Res. 33: 1162-1168. doi: 10.1093/nar/gki239.

Liang, H. and Landweber, L. 2007. Hypothesis: RNA editing of microRNA target sites in humans? RNA 13: 463-467.

Luciano, D.J., Mirsky, H., Vendetti, N.J., and Maas, S. 2004. RNA editing of a miRNA precursor. RNA 10: 1174-1177.

Maas, S., Rich, A., and Nishikura, K. 2003. A-to-I RNA editing: Recent news and residual mysteries. J. Biol. Chem. 278: 1391-1394.

Melcher, T., Maas, S., Herb, A., Sprengel, R., Higuchi, M., and Seeburg, P.H. 1996a. RED2, a brain specific member of the RNA- specific adenosine deaminase family. J. Biol. Chem. 271: 3179531798.

Melcher, T., Maas, S., Herb, A., Sprengel, R., Seeburg, P.H., and Higuchi, M. 1996b. A mammalian RNA editing enzyme. Nature 379: 460-464.

Neeman, Y., Levanon, E., Jantsch, M., and Eisenberg, E. 2006. RNA editing level in the mouse is determined by the genomic repeat repertoire. RNA 12: 1802-1809.

O’Connell, M., Krause, S., Higuchi, M., Hsuan, J., Totty, N., Jenny, A., and Keller, W. 1995. Cloning of cDNAs encoding mammalian double-stranded RNA-specific adenosine deaminase. Mol. Cell. Biol. 15: 1389-1397.

Polson, A. and Bass, B. 1994. Preferential selection of adenosines for modification by double-stranded RNA adenosine deaminase. EMBO J. 13: 5701-5711.

Popowicz, G., Schleicher, M., Noegel, A., and Holak, T. 2006. Filamins: Promiscuous organizers of the cytoskeleton. Trends Biochem. Sci. 31: 411-419.

Rae, F., Stephenson, S., Nicol, D., and Clements, J. 2000. Novel association of a diverse range of genes with renal cell carcinoma as identified by differential display. Int. J. Cancer 88: 726-732.

Robertson, S. 2005. Filamin A: Phenotypic diversity. Curr. Opin. Genet. Dev. 15: 301-307.

Scadden, A. and Smith, C. 2001. Specific cleavage of hyper-edited dsRNAs. EMBO J. 20: 4243-4252.

Smalheiser, N. and Torvik, V. 2006. Alu elements within human mRNAs are probable microRNA targets. Trends Genet. 22: 532-536.

$\mathrm{Su}$, A., Wiltshire, T., Batalov, S., Lapp, H., Ching, K., Block, D., Zhang, J., Soden, R., Hayakawa, M., Kreiman, G., et al. 2004. A gene atlas of the mouse and human protein-encoding transcriptomes. Proc. Natl. Acad. Sci. 101: 6062-6067.

Wang, Q., Miyakoda, M., Yang, W., Khillan, J., Stachura, D., Weiss, M., and Nishikura, K. 2004. Stress-induced apoptosis associated with null mutation of Adar1 RNA editing deaminase gene. J. Biol. Chem. 279: 4952-4961.

Wong, S., Sato, S., and Lazinski, D. 2001. Substrate recognition by Adar1 and Adar2. RNA 7: 846-858.

Yang, J., Sklar, P., Axel, R., and Maniatis, T. 1997. Purification and characterization of a human RNA adenosine deaminase for glutamate receptor B pre-mRNA editing. Proc. Natl. Acad. Sci. 94: 4354-4359.

Yang, W., Chendrimada, T., Wang, Q., Higuchi, M., Seeburg, P., Shiekhattar, R., and Nishikura, K. 2006. Modulation of microRNA processing and expression through RNA editing by ADAR deaminases. Nat. Struct. Mol. Biol. 13: 13-21.

Zhang, Z. and Carmichael, G. 2001. The fate of dsRNA in the nucleus: A p54(nrb)-containing complex mediates the nuclear retention of promiscuously A-to-I edited RNAs. Cell 106: 465-475. 

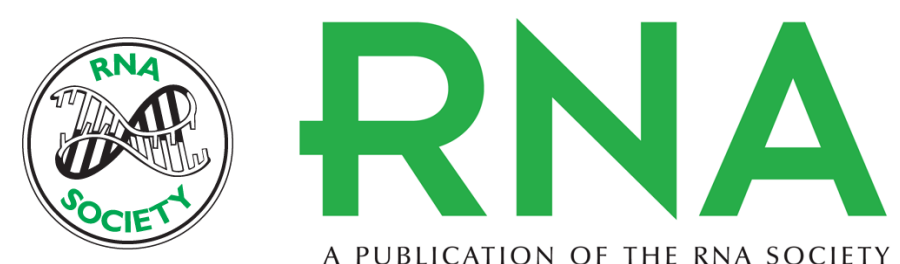

A PUBLICATION OF THE RNA SOCIETY

\section{Specificity of ADAR-mediated RNA editing in newly identified targets}

Eva M. Riedmann, Sandy Schopoff, Jochen C. Hartner, et al.

RNA 2008 14: 1110-1118

Supplemental
Material http://rnajournal.cshlp.org/content/suppl/2008/04/22/rna.923308.DC1

References This article cites 37 articles, 16 of which can be accessed free at: http://rnajournal.cshlp.org/content/14/6/1110.full.html\#ref-list-1

License

Email Alerting Receive free email alerts when new articles cite this article - sign up in the box at the Service top right corner of the article or click here. 